\title{
The QCD Phase Transition in a Strong Magnetic Background
}

\author{
Massimo D'Elia \\ Dipartimento di Fisica, Università di Genova and INFN, Via Dodecaneso 33, 16146 Genova, \\ Italy. \\ E-mail: massimo.delia@ge.infn.it
}

\section{Swagato Mukherjee}

Physics Department, Brookhaven National Laboratory, Upton, NY 11973-5000, USA

E-mail: swagato@quark.phy.bnl.gov

\section{Francesco Sanfilippo*}

Dipartimento di Fisica, Università di Roma "La Sapienza" and INFN, Sezione di Roma, Piazzale A. Moro 5, 00185 Roma, Italy

E-mail: Francesco.Sanfilippo@roma1.infn.it

We investigate the properties of the deconfining/chiral restoring transition for two flavor QCD in presence of a uniform background magnetic field. We adopt a standard staggered discretization of the fermion action, different values of the bare quark mass corresponding to pion masses ranging from 200 to $500 \mathrm{MeV}$, and magnetic fields up to $\mathrm{eB} \sim 1 \mathrm{GeV}^{2}$. We present first results regarding the dependence of the deconfinement and chiral transition temperature, as well as the strength of the transition on the magnetic field.

The XXVIII International Symposium on Lattice Field Theory

June 14-19,2010

Villasimius, Sardinia Italy

\footnotetext{
*Speaker.
} 


\section{Introduction}

The study of strong interactions in presence of background magnetic fields is relevant to many phenomenological contexts. Large magnetic fields $\left(B \sim 10^{16}\right.$ Tesla, i.e. $\left.\sqrt{|e| B} \sim 1.5 \mathrm{GeV}\right)$ may have been produced at the cosmological electroweak phase transition [1] and they may have influenced the QCD confinement/deconfinement and chiral restoring transition. Lower fields are expected to be produced in non-central heavy ion collisions, reaching up to $10^{14} \mathrm{~T}$ at RHIC and up to $10^{15} \mathrm{~T}$ at LHC $[2,3]$. Fields of the order of $10^{10} \mathrm{~T}$ are also expected in magnetars [4].

The influence of electric and magnetic fields on the chiral properties of the vacuum has been studied since some time, using various approximations or effective models of QCD $[5,6,7,8,10$, 11, 12]: the common feature of these studies are an enhancement of chiral symmetry breaking as a magnetic field is switched on. An important issue is also the influence of the magnetic field on the structure of the QCD phase diagram, in particular on the location and the nature of deconfinement and chiral symmetry restoring crossover. Clarifying that may be important to correctly predict the phenomenological consequences of the QCD transition on the evolution of the Universe during its early stages. Some computations exist, based on different approximations and QCD-like models $[13,14,15,16,17,18,19]$, which predict the possibility of a quite rich phenomenology, ranging from a possible splitting of deconfinement and chiral symmetry restoration to a sizable increase in the strength of the transition. However, the various model predictions are not always consistent among themselves.

Some indications to clarify these issues may come from first principle lattice QCD computations. Contrary to the case of a finite baryon density or of a background electric field, no technical difficulties hinder the numerical simulation of QCD in presence of a magnetic background field and systematic studies are possible. An investigation of the phase diagram in presence of a chromomagnetic background field has been performed in Refs. [20, 21], where it has been shown that the transition temperature decreases as a function of the chromomagnetic field, with deconfinement and chiral symmetry restoration remaining strictly related to each other. Lattice investigations in presence of an electro-magnetic background field have been done since long with the purpose of studying the magnetic properties of hadrons [22, 23]; recent studies [24, 25, 26, 27] have investigated mostly the chiral properties of the theory and the chiral magnetic effect.

We report on an investigation of the QCD phase transition in presence of an (electro-) magnetic background field. In particular we have considered $N_{f}=2$ QCD in the standard staggered discretization of the theory, and different values of the quark masses, in order to appreciate how the effects of the magnetic field change as the mass spectrum changes (in the heavy quark limit the magnetic field becomes irrelevant). A complete account of our results is reported in Ref. [28].

\section{Numerical Setup}

We shall consider two flavor QCD, with the up and down quarks carrying different electric charges and additionaly coupled to a background magnetic field. The presence of a background electro-magnetic field affects the propagation of dynamical quarks and that corresponds to a modification of the Dirac operator. In the continuum the covariant derivative changes by inclusion of the electro-magnetic $A_{\mu}$ field; on the lattice one has to add appropriate $U(1)$ fields to the gauge 
link variables appearing in the fermion matrix which parallel transport quark fields from one lattice site to the other. In the case of a uniform background magnetic field $B$, with two different electric charges for the two quark flavors, $q_{u}=2|e| / 3$ and $q_{d}=-|e| / 3(|e|$ being the elementary electric charge), the partition function of the (rooted) staggered fermion discretized version of the theory is

$$
\begin{aligned}
Z(T, B) & \equiv \int \mathscr{D} U e^{-S_{G}} \operatorname{det} M^{\frac{1}{4}}\left[B, q_{u}\right] \operatorname{det} M^{\frac{1}{4}}\left[B, q_{d}\right], \text { where } \\
M_{i, j}[B, q] & =a m \delta_{i, j}+\frac{1}{2} \sum_{v=1}^{4} \eta_{i, v}\left(u(B, q)_{i, v} U_{i, v} \delta_{i, j-\hat{v}}-u^{*}(B, q)_{i-\hat{v}, v} U_{i-\hat{v}, v}^{\dagger} \delta_{i, j+\hat{v}}\right) .
\end{aligned}
$$

$\mathscr{D} U$ denotes the functional integration over the $S U(3)$ gauge link variables $U_{n, \mu}, S_{G}$ is the discretized pure gauge action (we consider a standard Wilson plaquette action). The subscripts $i$ and $j$ refer to lattice sites, $\hat{v}$ is a unit vector on the lattice and $\eta_{i, v}$ are the staggered phases. Periodic (antiperiodic) boundary conditions must be taken for gauge (fermion) fields along the Euclidean time direction, while in the spatial directions periodic boundary conditions will be chosen for all fields. $u(B, q)_{i, v}$ are the gauge links corresponding to the background $U(1)$ magnetic field. We shall consider a constant magnetic field $\vec{B}=B \hat{z}$ and the following choice for the gauge field:

$$
A_{y}=B x ; \quad A_{\mu}=0 \text { for } \mu=x, z, t .
$$

That corresponds to the following choice for the lattice $U(1)$ links:

$$
u(B, q)_{n, y}=e^{i a^{2} q B n_{x}} ; \quad u(B, q)_{n, \mu}=1 \text { for } \mu=x, z, t
$$

This choice corresponds to a magnetic flux $a^{2} B$ going through each plaquette in the $x-y$ plane, except at the boundary $\left(L_{x}, y, z, t\right)$ due to the presence of periodic boundary conditions along the spatial directions. In order to guarantee the smoothness of the background field across and gauge invariance, the $U(1)$ gauge fields must be modified at the boundary of the $x$ direction [29]:

$$
u(B, q)_{n, x=L_{x}}=e^{-i a^{2} q L_{x} B n_{y}} .
$$

It is easy to check that the magnetic flux through all the plaquettes in the priodic $x-y$ plane remains constant only if one uses a quantized magnetic field $-a^{2} q B=2 \pi b / L_{x} L_{y}$, where $b$ is an integer. The quantization of $B$ in our case is set by the $d$ quark charge $q_{d}=-|e| / 3$,

$$
|e| B=6 \pi T^{2}\left(\frac{N_{t}}{L_{s}}\right)^{2} b,
$$

$T=1 /\left(N_{t} a\right)$ is the temperature and $L_{x}=L_{y} \equiv L_{s}$.

Our simulations have been carried out on $16^{3} \times 4$ lattices. We have done simulations for three different sets of bare quark masses $a m=0.01335,0.025$ and 0.075 . The corresponding (Goldstone) pion masses are $a m_{\pi}=0.307(3), 0.417(3)$ and $0.707(3)$. The temperature $T=1 /\left(N_{t} a\right)$ is changed by varying the lattice spacing through the inverse gauge coupling $\beta$.

$T=0$ estimates of the string tension, done at the same $\beta$ values where the transition at zero magnetic field takes place, lead to estimate $a$ ranging from 0.29 to $0.31 \mathrm{fm}$ as the quark mass is decreased, corresponding to $T_{c}(B=0)$ ranging from 170 to $160 \mathrm{MeV}$. The corresponding physical 

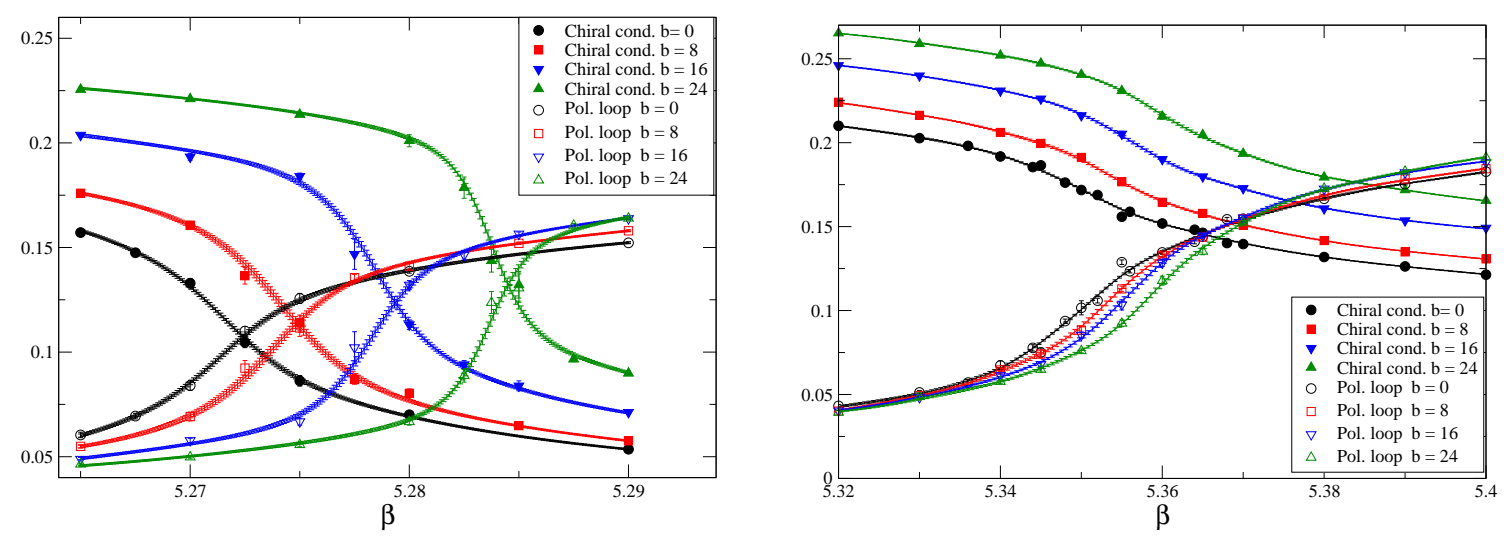

Figure 1: Chiral condensate and Polyakov loop for $a m=0.01335$ (left) and $a m=0.075$ (right).

values of the (Goldstone) pion mass are $m_{\pi} \approx 195,275$ and $480 \mathrm{MeV}$. For each of these pion masses we have done simulations using magnetic fields corresponding to $b=0,8,16$ and 24, i.e. for $|e| B=0,3 \pi T^{2}, 6 \pi T^{2}$ and $9 \pi T^{2}$. Thus, for the lightest quark mass and taking the actual pion mass used in our simulations as a reference, our magnetic field reaches values up to about $|e| B \approx 19 m_{\pi}^{2}$, which corresponds to $\sqrt{|e| B} \approx 850 \mathrm{MeV}$ in physical units. Note that, since we are working with a fixed value of $N_{t}$, the magnetic field changes with changing temperature as $a^{-2} \propto T^{2}$. However, for all the quark masses the range of coupling, i.e. the range of $a$, that we explore corresponds to a $<2 \%$ change in $T$ and hence the magnetic field only changes at most by a few percent.

We have made use of an RHMC algorithm to simulate rooted staggered fermions: even if we have two flavors, we need to treat separately each of them and thus take the fourth root of the fermion determinant. Typical statistics are of the order of 10k molecular dynamics trajectories.

\section{Numerical Results}

In Fig. 1 we show the behaviour of the quark condensate (average of $u$ and $d$ condensates) and of the Polyakov loop as a function of the magnetic field, for $a m=0.01335$ in the left panel, and for $a m=0.075$ in the right one. Results are presented as a function of the inverse gauge coupling $\beta$.

The quark condensate increases as a function of the magnetic field independently of $T$, as expected from various analytic predictions. If we interpret the drop of the condensate as the signal for chiral symmetry restoration, then the transition temperature increases as a function of the magnetic field and, especially for the lowest quark masses, a much sharper drop is observed for the highest magnetic fields explored, indicating a sizable increase in the strength of the transition. The increase in the strength of the transition is also clearly visible from the behaviour of the disconnected chiral susceptibility, which is shown in right panel of Fig. 2 for the lowest quark mass.

Regarding the Polyakov loop, from Fig. 1 we see that, while in the low temperature regime it decreases as a function of $B$ (as one would expect qualitatively from the fact that the chiral condensate increases), at high $T$ it increases. Such behaviour should be better understood from a 

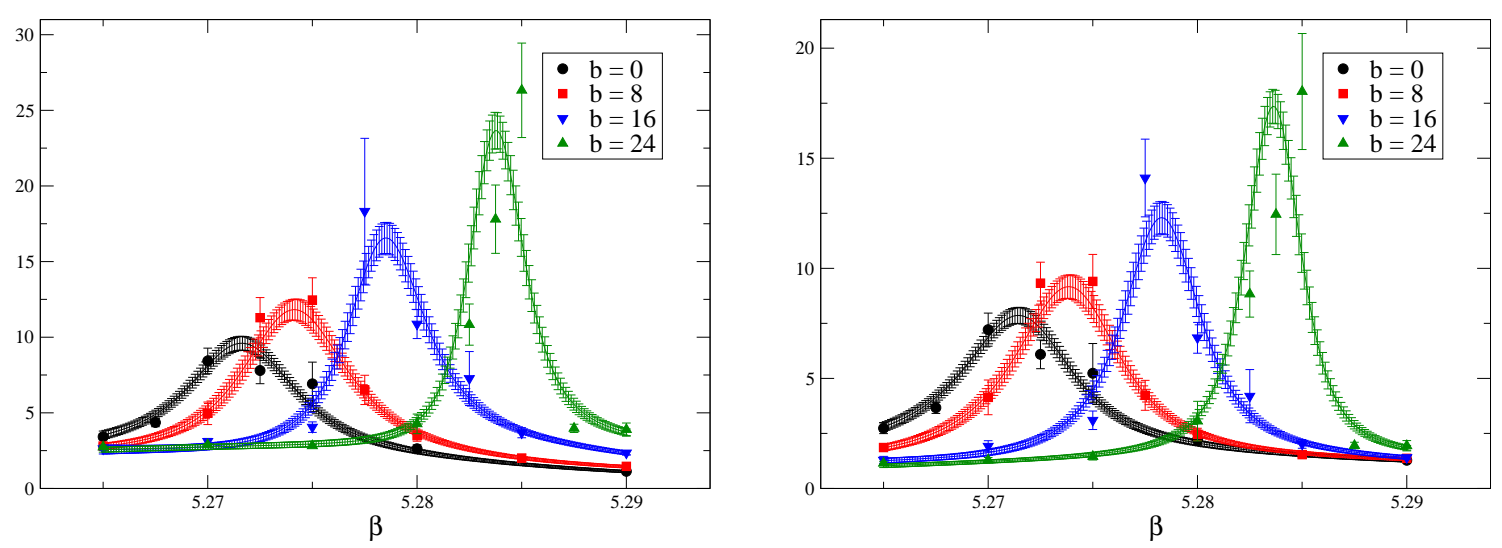

Figure 2: Disconnected $\langle\bar{\psi} \psi\rangle$ susceptibility (left) and Polyakov loop susceptibility (right) for am $=0.01335$.

theoretical point of view, however we notice that a qualitatively similar behaviour can be observed in the data reported in Ref. [17], which were obtained by a PNJL model analysis.

If we interpret the rise of the Polyakov loop as the onset of deconfinement, then we infer that the shift and the increase in strength of the deconfinement transition is in agreement with what observed for the chiral transition. Data obtained for the Polyakov loop susceptibility, which are shown left panel of Fig. 2 for the lowest quark mass explored, lead to similar conclusions.

In Table 1 we report the pseudocritical couplings $\beta_{c}$ for deconfinement and chiral restoration obtained by fitting the peak of the susceptibilities by a quadratic function. We have also determined $\beta_{c}$ looking for the inflection point of observables, by means of polynomial fits, obtaining compatible results. Data obtained for the pseudocritical couplings confirm what is already visible at a qualitative level from Figs. 1: no appreciable separation of chiral restoration and deconfinement is induced by the presence of the background field, at least for the explored field strengths.

From the values of the pseudocritical couplings we obtain the ratio $T_{c}(B) / T_{c}(0)$ as a function of the dimensionless ratio $e B / T^{2}$, as reported in Fig. 3 (left). We have made use of the 2-loop $\beta$-function to convert the information on $\beta_{c}$ in a ratio of physical temperatures.

From Fig. 3 we learn that the change in temperature is pretty small and of the order of a few percent at the highest explored fields. Moreover, there seems to be a saturation as the chiral limit is approached and results for $a m=0.01335$ and $a m=0.025$ stay onto each other. Notice, however, that this is true if we plot results as a function of $|e| B / T^{2}$ : had we used $|e| B / m_{\pi}^{2}$ results would have been much different for the different masses: the highest magnetic field is about $20 m_{\pi}^{2}$ for the lowest mass and about $10 m_{\pi}^{2}$ for the intermediate mass. This suggests that, at least for the strong fields and for the pion masses used in our simulations, the relevant scale which governs the effect of the magnetic field on the shift of the transition is the physical temperature itself and not $m_{\pi}$.

We have also tried to understand what is the functional dependence of $T_{c}(B)$ on the magnetic field and fitted our data for the lowest mass according to

$$
\frac{T_{c}(B)}{T_{c}(0)}=1+A\left(\frac{|e| B}{T^{2}}\right)^{\alpha}
$$




\begin{tabular}{|c||c|c||c|c||c|c|}
\hline$b$ & $\beta_{c}^{\text {Pol }}(0.01335)$ & $\beta_{c}^{\psi \psi}(0.0)$ & $\beta_{c}^{\text {Pol }}(0.025)$ & $\beta_{c}^{\psi \psi}(0.025)$ & $\beta_{c}^{\text {Pol }}(0.075)$ & $\beta_{c}^{\psi \psi}(0.075)$ \\
\hline 0 & $5.2714(4)$ & $5.2716(3)$ & $5.2893(2)$ & $5.2898(3)$ & $5.351(1)$ & $5.351(2)$ \\
8 & $5.2739(4)$ & $5.2741(4)$ & $5.2925(3)$ & $5.2925(3)$ & $5.353(1)$ & $5.353(2)$ \\
16 & $5.2783(3)$ & $5.2785(3)$ & $5.2961(3)$ & $5.2966(3)$ & $5.355(1)$ & $5.357(2)$ \\
24 & $5.2836(2)$ & $5.2838(2)$ & $5.3014(4)$ & $5.3018(4$ & $5.358(1)$ & $5.360(1)$ \\
\hline
\end{tabular}

Table 1: Pseudocritical couplings from fit of the peak of chiral condensate or Polyakov loop susceptibilities.
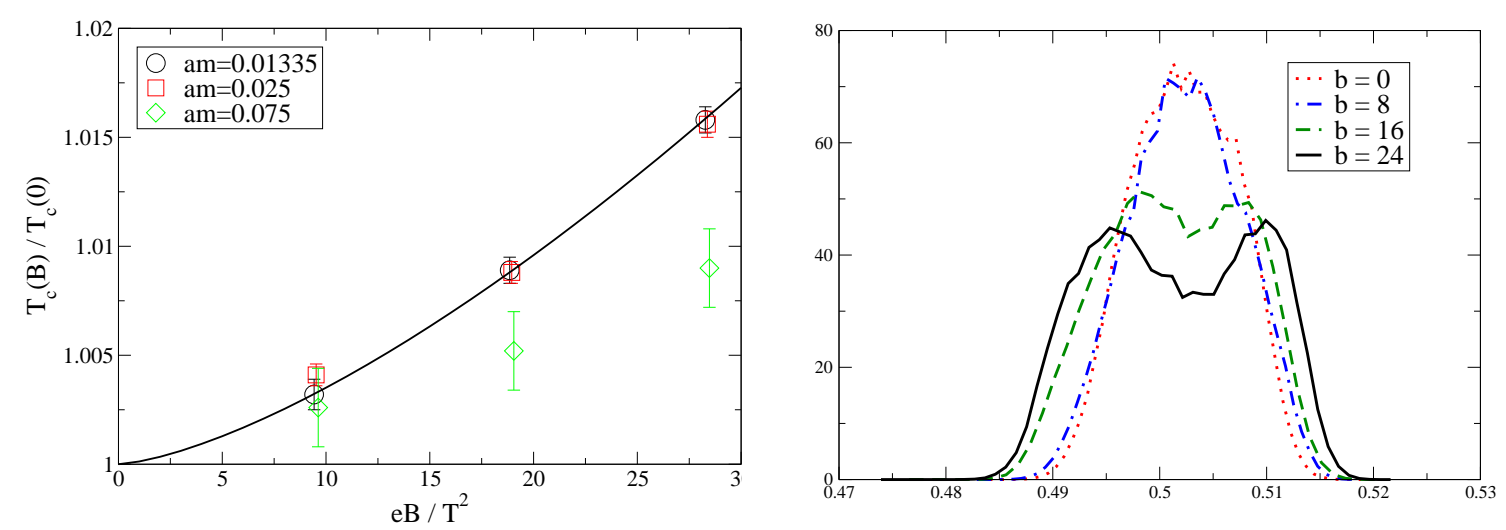

Figure 3: Left: $T_{c}(B)$ for different quark masses. The solid curve is a power law fit to the lightest quark data (see text). Right: Reweighted plaquette distribution at $\beta_{c}$ as a function of the external field at $a m=0.01335$ on a $16^{3} \times 4$ lattice.

finding that $\alpha=1.45(20)$ and $A \sim 1.310^{-4}$. The fit is reported in Fig. 3 as well.

Finally we discuss the nature of the deconfinement/chiral restoring transition. At $B=0$ it is still unclear whether a weak first order transition may be present in the chiral limit [30,31], however no clear signal of metastability or of finite latent heat has still been detected at finite quark masses and on available lattice sizes: the first order transition, even if present, is extremely weak, hence of poor phenomenological relevance. On the other hand our results show that the introduction of a magnetic field makes the transition sharper. The question is whether a large enough field can turn the transition into a first order strong enough to become phenomenologically relevant and also become visible on realistic volumes used in lattice simulations. To that aim we have analyzed the reweighted plaquette distribution at the critical couplings and for different values of the magnetic field: results are shown in Fig. 3 (right). The single peak distribution which is present at zero or small magnetic field turns into a double peak distribution, typical of a first order transition, for the largest magnetic field explored. We consider that only as a preliminary indication: numerical simulations on larger lattice sizes are necessary to make a proper finite size scaling analysis.

\section{Acknowledgments}

S. M. is supported under Contract No. DE-AC02-98CH10886 with the U.S. Department of Energy. 


\section{References}

[1] T. Vachaspati, Phys. Lett. B 265, 258 (1991).

[2] D. E. Kharzeev, L. D. McLerran and H. J. Warringa, Nucl. Phys. A 803, 227 (2008).

[3] V. Skokov, A. Y. Illarionov and V. Toneev, Int. J. Mod. Phys. A 24, 5925 (2009).

[4] R. C. Duncan and C. Thompson, Astrophys. J. 392, L9 (1992).

[5] S. P. Klevansky and R. H. Lemmer, Phys. Rev. D 39, 3478 (1989).

[6] H. Suganuma and T. Tatsumi, Annals Phys. 208, 470 (1991).

[7] V. P. Gusynin, V. A. Miransky and I. A. Shovkovy, Phys. Lett. B 349, 477 (1995).

[8] I. A. Shushpanov and A. V. Smilga, Phys. Lett. B 402, 351 (1997).

[9] D. Ebert, K. G. Klimenko, M. A. Vdovichenko and A. S. Vshivtsev, Phys. Rev. D 61, 025005 (2000).

[10] V. A. Miransky and I. A. Shovkovy, Phys. Rev. D 66, 045006 (2002).

[11] T. D. Cohen, D. A. McGady and E. S. Werbos, Phys. Rev. C 76, 055201 (2007).

[12] A. V. Zayakin, JHEP 0807, 116 (2008).

[13] K. G. Klimenko, Z. Phys. C 54, 323 (1992).

[14] N. O. Agasian and S. M. Fedorov, Phys. Lett. B 663, 445 (2008).

[15] E. S. Fraga and A. J. Mizher, Phys. Rev. D 78, 025016 (2008);

[16] J. K. Boomsma and D. Boer, Phys. Rev. D 81, 074005 (2010).

[17] K. Fukushima, M. Ruggieri and R. Gatto, arXiv:1003.0047 [hep-ph].

[18] A. J. Mizher, M. N. Chernodub and E. S. Fraga, arXiv:1004.2712 [hep-ph].

[19] R. Gatto and M. Ruggieri, arXiv:1007.0790 [hep-ph].

[20] P. Cea and L. Cosmai, JHEP 0508, 079 (2005).

[21] P. Cea, L. Cosmai and M. D’Elia, JHEP 0712, 097 (2007).

[22] G. Martinelli, G. Parisi, R. Petronzio and F. Rapuano, Phys. Lett. B 116, 434 (1982).

[23] C. W. Bernard, T. Draper, K. Olynyk and M. Rushton, Phys. Rev. Lett. 49, 1076 (1982).

[24] P. V. Buividovich, M. N. Chernodub, E. V. Luschevskaya and M. I. Polikarpov, Phys. Rev. D 80, 054503 (2009).

[25] M. Abramczyk, T. Blum, G. Petropoulos and R. Zhou, arXiv:0911.1348 [hep-lat].

[26] P. V. Buividovich, M. N. Chernodub, E. V. Luschevskaya and M. I. Polikarpov, Phys. Lett. B 682, 484 (2010), Nucl. Phys. B 826, 313 (2010).

[27] P. V. Buividovich et al., arXiv:1003.2180 [hep-lat].

[28] M. D’Elia, S. Mukherjee and F. Sanfilippo, Phys. Rev. D 82, 051501 (2010)

[29] M. H. Al-Hashimi and U. J. Wiese, Annals Phys. 324, 343 (2009).

[30] M. D’Elia, A. Di Giacomo and C. Pica, Phys. Rev. D 72, 114510 (2005).

[31] G. Cossu, M. D’Elia, A. Di Giacomo and C. Pica, arXiv:0706.4470 [hep-lat]. 